\section{Effect of Aminoethoxyvinylglycine and Surfactants on Preharvest Drop, Maturity, and Fruit Quality of Two Processing Peach Cultivars}

\author{
J.A. Cline \\ University of Guelph, Department of Plant Agriculture, Horticultural \\ Experiment Station, Simcoe, Ontario N3Y 4N5 Canada \\ Additional key words. Prunus, AVG, fruit quality, adjuvants
}

\begin{abstract}
The effect of aminoethoxyvinylglycine (AVG), commercially available as ReTain, and three organo-silicone surfactants were evaluated in a series of four experiments over a 2-year period in two commercial peach orchards. Four rates of AVG $(0,66,132$, and 264 $\mathrm{mg} \cdot \mathrm{L}^{-1} \mathrm{AVG}$; all applied with $0.05 \%$ Sylgard 309$)$ and three surfactants $(0.05 \%$ Sylgard $309 ; 0.05 \%$ Regulaid; and $0.50 \%$ LI-700; all applied with $132 \mathrm{mg} \cdot \mathrm{L}^{-1} \mathrm{AVG}$ ) were applied to 'Venture' and 'Babygold 7' peach trees 10 days before first harvest. Fruit were harvested according to commercial standard maturation criteria of background color, suture filling, and fruit size. Treatments were assessed in relation to fruit maturity, delay in harvest, fruit size and yield, fruit quality (flesh firmness and brix), as well as fruit quality following 2 weeks of cold storage. Based on sequential harvest data, the maturation of the AVG treated trees was delayed by about 3 to 4 days. Fruit from AVG treated trees were firmer at harvest and 2 weeks following cold storage at $2^{\circ} \mathrm{C}$. However, no additional increase in fruit size or yield was detected. In addition, the addition of a surfactant was not necessary for AVG to be efficacious for delaying maturity and enhancing firmness when applied at $132 \mathrm{mg} \cdot \mathrm{L}^{-1}$ AVG. However, when the three surfactants were compared, Regulaid and $\mathrm{Li}$ 700 advanced color development in one experiment and Li-700 resulted in firmer fruit in another. Aminoethoxyvinylglycine applications to the clingstone cultivars 'Venture' and 'Babygold 7' can be used successfully to manage harvest activities by delaying the onset of picking and improving fruit firmness.
\end{abstract}

Fruit softening and other ethylene-mediated processes in peach are correlated with an increase in 1-amino-cyclopropane-carboxylate (ACC) synthase (Tonutti et al., 1977). Aminoethoxyvinylglycine $(\mathrm{AVG})$ is a plant growth bio-substance known to competitively inhibit ACC synthase, a rate limiting enzyme in the ethylene biosynthesis pathway. Research on ReTain (Valent BioSciences, Chicago, Ill.), a commercial formulation of AVG $(15 \% \mathrm{w} / \mathrm{w})$, was developed and first registered for use on apples (Autio and Bramlage, 1982; Bangerth, 1978; Greene, 2002; Greene and Schupp, 2004; Schupp and Greene, 2004). The merits of using AVG on other climacteric fruit, such as peaches, is not well understood. Nonmelting processing peach cultivars, grown principally for the processing industry, would benefit from the same advantages that AVG offers for apples - a compressed harvest window for multiple-pick cultivars, improved firmness, storage potential, fruit size, fruit removal force, and reduced preharvest fruit drop. In addition, if preharvest softening of peaches could be delayed, it not only could provide growers more time to

Received for publication 5 Aug. 2005. Accepted for publication 11 Nov. 2005. We gratefully acknowledge the support of the Ontario Ministry of Agriculture, Food and Rural Affairs and Valent BioSciences for partial funding of this project, John Fedorkow and John Smith for providing use of their orchards, Rocco Guaranccia and Debbie Norton for assisting with data collection and Robert Cline for reviewing this manuscript. harvest but also might allow additional fruit growth and reduced physical damage.

In previous research on 'Redhaven' peach trees, AVG applied at $500 \mathrm{mg} \cdot \mathrm{L}^{-1}$ at the end of stage I to midstage III of fruit growth had little effect on fruit maturity and fruit quality (Byers, 1997). However, in another study on 'Mibaekdo' peach (Kim et al., 2004), AVG applied at rates ranging from 100 to $200 \mathrm{mg} \cdot \mathrm{L}^{-1}$, 21 to $28 \mathrm{~d}$ before first harvest delayed fruit maturity by about $3 \mathrm{~d}$ and reduced preharvest fruit drop. In addition, Vizzotto et al. (2002) found that $\mathrm{AVG}$, applied at rates ranging from 62.5 to $250 \mathrm{mg} \cdot \mathrm{L}^{-1} 10 \mathrm{~d}$ before harvest to 'Redhaven' peach trees, delayed the onset of fruit ethylene evolution, reduced fruit drop, delayed fruit softening, and slightly increased soluble solids. Furthermore, Singh et al (2003) found that when AVG, applied at $125 \mathrm{mg} \cdot \mathrm{L}^{-1}, 5$ to $15 \mathrm{~d}$ before harvest to several fresh market cultivars, extended the harvest period, delayed fruit maturity, increased fruit firmness, and total soluble solids and in several, but not all cultivars. AVG has also been used successfully as a postharvest dip to delay softening and enhance the shelf-life of peaches (Byers, 1997; Garner et al., 2001). The literature overall indicates that for both apple and peach, the effects of AVG are influenced by concentration, time application with respect to harvest, and with cultivar (Autio and Bramlage, 1982).

The objectives of this study were to measure the fruit quality and yield benefits of AVG applied at various rates $(0,66,132$, and 264 $\mathrm{mg} \cdot \mathrm{L}^{-1}$ ), and to compare three organo-silicone based surfactants (Sylgard 309; Regulaid; and LI-700) at a single rate of AVG, on 'Venture' and 'Babygold 7' peaches, two processing cultivars grown commercially in the Niagara Peninsula. Information on the benefits of AVG on these two processing cultivars is lacking, as is efficacy data on surfactants that are registered in Canada and that could be potentially be used with the commercial formulation, ReTain.

\section{Materials and Methods}

Experiment 1. A commercial block of 4-yrold 'Venture' peach trees grafted on 'Bailey' rootstock and located in St. David's, Ont., was used for this study. Trees were spaced at $3.7 \times$ $4.5 \mathrm{~m}$ (500 trees/ha) and trained to a free-standing central leader, without irrigation. Standard commercial orchard management practices for the region were used (OMAF, 2004). Four rates of AVG were applied by handgun to single trees using a research sprayer set at $1379 \mathrm{kPa}$. To minimize spray drift, experimental units were separated by at least one guard tree. Treatments consisted of a) water, no AVG; b) $66 \mathrm{mg} \cdot \mathrm{L}^{-1}$ AVG; c) $132 \mathrm{mg} \cdot \mathrm{L}^{-1} \mathrm{AVG}, 264 \mathrm{mg} \cdot \mathrm{L}^{-1} \mathrm{AVG}$. All treatments including the control, contained 0.05\% Sylgard 309 nonionic organo-silicone surfactant (Dow Corning Canada Inc, Toronto) and were applied on 31 Aug. 2002, $10 \mathrm{~d}$ before the anticipated first harvest date.

For Expts. 1 and 2, the number of dropped fruit were counted on 4, 6, 9, 12, and 16 Sept. 2002. Fruit were harvested, weighed and counted on 6,9, 12, and 16 Sept. based on similar visual background color. On each harvest date, fruit quality (fruit weight, firmness, soluble solids, percent surface red color, ground and blush color) was measured on 10 unblemished, sound fruit. On 9 and 12 Sept., 10 unblemished, sound fruit were also randomly collected and stored at $2{ }^{\circ} \mathrm{C}$ for 2 weeks to determine treatments effects on fruit storage potential. At the end of the growing season, trunk circumference was measured and trunk cross sectional area and crop load calculated.

Experiment 2. In the same commercial block as Expt. 1 (same cultivar, spacing, tree age) three spray surfactants were compared at the common rate of $132 \mathrm{mg} \cdot \mathrm{L}^{-1}$ AVG. The surfactant treatments were applied by handgun using the same methods as Experiment 1. Treatments consisted of a) $132 \mathrm{mg} \cdot \mathrm{L}^{-1} \mathrm{AVG}$, no surfactant; b) $132 \mathrm{mg} \cdot \mathrm{L}^{-1}$ AVG plus $0.05 \%$ Sylgard 309; c) $132 \mathrm{mg} \cdot \mathrm{L}^{-1}$ AVG plus $0.05 \%$ Regulaid (Kalo Inc, Overland Park, KS), and; 4) $132 \mathrm{mg} \cdot \mathrm{L}^{-1} \mathrm{AVG}$. and $0.50 \% \mathrm{LI}-700$ (Loveland Industries, Greeley, Colo.), were replicated five times, and were applied on 31 Aug. 2002. Fruit drop, yield, and quality data recorded were identical to Expt. 1.

Experiment 3. In 2003, a commercial block of 7-year-old 'Venture' peach trees grafted on 'Bailey' rootstock located in Vineland was used for this study. Trees were spaced $3.4 \times$ $5.5 \mathrm{~m}$, were not trickle irrigated and trained to a free-standing central leader.

A randomized complete block design was used with seven treatments and four single-tree replications. Experimental units were separated 
by a guard tree on each side to minimize spray drift. Treatments consisted of 1) surfactant only; 2) $66 \mathrm{mg} \cdot \mathrm{L}^{-1}+0.05 \%$ Sylgard 309 ; 3) $132 \mathrm{mg} \cdot \mathrm{L}^{-1}+0.05 \%$ Sylgard 309 ; 4) 264 $\mathrm{mg} \cdot \mathrm{L}^{-1}+0.05 \%$ Sylgard $\left.309 ; 5\right) 132 \mathrm{mg} \cdot \mathrm{L}^{-1}+$

no surfactant; 6) $\mathrm{mg} \cdot \mathrm{L}^{-1}+0.05 \%$ Regulaid; and 7) $132 \mathrm{mg} \cdot \mathrm{L}^{-1}+0.5 \% \mathrm{Li}-700$. Spray treatments were applied 3 Sept. using the same methods described in Expt. 1. At each of five successive dates $(10,15,18,22$, and 24 Sept.) naturally

Table 1. Effect of AVG rate and various surfactants on yield components and trunk cross-sectional area of 'Venture' peaches in 2002.

\begin{tabular}{|c|c|c|c|c|}
\hline Treatment & $\begin{array}{c}\text { Total } \\
\text { harvested } \\
\text { fruit/tree } \\
\text { (no.) }\end{array}$ & $\begin{array}{c}\text { Total } \\
\text { yield } \\
(\mathrm{kg} / \text { tree })\end{array}$ & $\begin{array}{c}\text { Crop } \\
\text { load } \\
\text { (no. fruit } / \mathrm{cm}^{2} \text { ) }\end{array}$ & $\begin{array}{c}\text { Trunk } \\
\text { cross-sectional } \\
\text { area } \\
\left(\mathrm{cm}^{2}\right)\end{array}$ \\
\hline \multicolumn{5}{|c|}{ Experiment 1-Rate of ReTain ${ }^{z}$} \\
\hline 0 mg.L-1 & 59.4 & 9.3 & 2.3 & 30.9 \\
\hline 66 mg.L-1 & 61.0 & 10.3 & 2.6 & 28.6 \\
\hline 132 mg.L-1 & 54.8 & 9.1 & 2.3 & 27.8 \\
\hline 264 mg.L-1 & 51.6 & 8.4 & 2.2 & 26.7 \\
\hline Signifigancez & NS & NS & NS & NS \\
\hline $\operatorname{LSD}(p=0.05)$ & 33.17 & 5.58 & 1.1 & 5.10 \\
\hline$P$ & 0.908 & 0.903 & 0.9 & 0.368 \\
\hline \multicolumn{5}{|c|}{ Experiment $2-$ Surfactant $^{\mathrm{y}}$} \\
\hline None & 57.8 & 10.0 & 2.3 & 30.5 \\
\hline $0.05 \%$ Sylgard 309 & 65.8 & 11.4 & 2.8 & 29.4 \\
\hline $0.05 \%$ Regulaid & 63.5 & 10.7 & 2.5 & 31.2 \\
\hline $0.50 \%$ LI 700 & 64.8 & 11.6 & 2.3 & 33.4 \\
\hline Signifigancez & NS & NS & NS & NS \\
\hline $\operatorname{LSD}(p=0.05)$ & 39.1 & 6.3 & 1.5 & 10.1 \\
\hline$P$ & 0.966 & 0.932 & 0.868 & 0.831 \\
\hline
\end{tabular}

${ }^{\mathrm{z}}$ All treatments in Expt. 1 include $0.05 \%$ Sylgard 309 surfactant.

y All treatments in Expt. 2 include $132 \mathrm{mg} \cdot \mathrm{L}^{-1}$ of Retain.

$\mathrm{NS}^{*, * * * * * *}$ Nonsignificant or signifigant differences at $P=0.05,0.01$, or 0.001 respectively. Mean separation within columns by LSD at $P=0.05$. abscised fruit were weighed and counted (and discarded). At each of four dates $(15,18,22$, and 24 Sept.), mature fruit were harvested on a uniform background color and their weight and number recorded. On each of the harvest dates, a random subsample of 10 fruit (free on split pits, insect damage, bruising, etc) were collected for fruit quality determination. On 18 and 22 Sept., 10 unblemished, sound fruit were also collected for their storage quality potential after 2 weeks in regular air storage $\left(2^{\circ} \mathrm{C}\right)$.

Experiment 4. In the same commercial orchard described in Expt. 3, a block of 6-yrold 'Babygold 7' peaches on Bailey seedling rootstock planted at a spacing of $2.7 \times 5.5 \mathrm{~m}$ was also used in this experiment. Cultural techniques, experimental design, and treatments were the same as those used in Expt. 3, with the exception that treatments were replicated five times. The first commercial harvest dates of 'Babygold 7' was 18 Sept.

Fruit quality. Surface blush color and ground color were measured on each 10 (Expts. 1 and 2) or 15 fruit (Expts. 3 and 4) sample using a tristimulus colorimeter (model CR300; Minolta, Toronto). Fruit chromaticity was recorded in the $\mathrm{L}^{*}, \mathrm{a}^{*}, \mathrm{~b}^{*}$ space coordinates and the Chroma $\mathrm{C}^{*}$ and hue angle $\left(\mathrm{h}^{\circ}\right)$ were calculated (McGuire, 1992). Flesh firmness was evaluated on each fruit using an electronic

Table 2. Effect of AVG rate and various surfactants on the percent of harvested fruit by date, mean fruit weight, and preharvest fruit drop of 'Venture' peaches in 2002.

\begin{tabular}{|c|c|c|c|c|c|c|c|c|c|c|c|c|c|c|}
\hline \multirow[b]{2}{*}{ Treatment } & \multicolumn{3}{|c|}{$\begin{array}{c}\text { Fruit harvested } \\
\text { (\% of total harvested by no.) }\end{array}$} & \multicolumn{3}{|c|}{$\begin{array}{l}\text { Cumulative fruit harvest } \\
\text { (\% of total harvested by no.) }\end{array}$} & \multicolumn{3}{|c|}{$\begin{array}{l}\text { Mean fruit wt } \\
\text { (g/fruit) }\end{array}$} & \multicolumn{5}{|c|}{$\begin{array}{l}\text { Cumulative fruit drop } \\
{[\% \text { dropped of total fruit no. }} \\
\text { (harvested+dropped)] }\end{array}$} \\
\hline & 9 Sept. & 12 Sept. & $16 \mathrm{Sept}$ & 9 Sept. & 12 Sept. & 16 Sept. & 9 Sept. & 12 Sept. & $16 \mathrm{Sept}$. & 4 Sept. & 6 Sept. & 9 Sept. & 12 Sept. & 16 Sept. \\
\hline \multicolumn{15}{|c|}{ Experiment 1 - Rate of ReTain ${ }^{2}$} \\
\hline $0 \mathrm{mg} \cdot \mathrm{L}^{-1}$ & 72 & 28 & 0 & 72 & 100 & 100 & 160.9 & 154.3 & --- & 6 & 9 & 14 & 19 & 18 \\
\hline $66 \mathrm{mg} \cdot \mathrm{L}^{-1}$ & 43 & 40 & 17 & 43 & 83 & 100 & 166.6 & 176.2 & 155.6 & 5 & 6 & 10 & 13 & 16 \\
\hline $132 \mathrm{mg} \cdot \mathrm{L}^{-1}$ & 49 & 32 & 19 & 49 & 81 & 100 & 157.8 & 178.8 & 158.1 & 5 & 7 & 11 & 14 & 16 \\
\hline $264 \mathrm{mg} \cdot \mathrm{L}^{-1}$ & 47 & 25 & 28 & 47 & 72 & 100 & 160.6 & 174.1 & 170.5 & 5 & 7 & 9 & 11 & 15 \\
\hline Signifigance & $*$ & NS & $*$ & $*$ & $*$ & -- & NS & NS & NS & NS & NS & NS & NS & NS \\
\hline $\operatorname{LSD}(p=0.05)$ & 19 & 15 & 17 & 19 & 17 & --- & 25.9 & 27.8 & 22.7 & 7.2 & 8.8 & 11.4 & 11.8 & 11.8 \\
\hline$P$ & 0 & 0 & 0 & 0 & 0 & --- & 0.9 & 0.3 & 0.3 & 1.0 & 0.9 & 0.8 & 0.5 & 0.9 \\
\hline \multicolumn{15}{|c|}{ Experiment $2-$ Surfactant $^{y}$} \\
\hline None & 42 & 27 & 31 & 42 & 69 & 100 & 174.6 & 167.0 & 174.0 & 6 & 6 & 7 & 10 & 14 \\
\hline $0.05 \%$ Sylgard 309 & 44 & 24 & 32 & 44 & 68 & 100 & 167.6 & 189.3 & 176.5 & 6 & 7 & 12 & 16 & 21 \\
\hline $0.05 \%$ Regulaid & 42 & 29 & 30 & 42 & 70 & 100 & 173.8 & 188.4 & 156.7 & 4 & 4 & 7 & 11 & 18 \\
\hline $0.50 \%$ LI 700 & 42 & 48 & 9 & 42 & 91 & 100 & 175.1 & 177.6 & 187.7 & 4 & 4 & 8 & 12 & 13 \\
\hline Signifigancez & NS & $* *$ & NS & NS & NS & --- & NS & NS & NS & NS & NS & NS & NS & NS \\
\hline $\operatorname{LSD}(p=0.05)$ & 22 & 13 & 26 & 22 & 26 & --- & 35.4 & 35.6 & 45.9 & 7.6 & 8.3 & 10.8 & 9.8 & 10.8 \\
\hline$P$ & 0.996 & 0.007 & 0.23 & 0.996 & 0.23 & --- & 0.959 & 0.482 & 0.521 & 0.92 & 0.885 & 0.677 & 0.589 & 0.36 \\
\hline
\end{tabular}

${ }^{\mathrm{z}}$ All treatments in Experiment 1 include $0.05 \%$ Sylgard 309 surfactant.

y All treatments in Experiment 2 include $132 \mathrm{mg} \cdot \mathrm{L}^{-1}$ of Retain.

NS,,$* * * * * * *$ Nonsignificant or signifigant differences at $P=0.05,0.01$, or 0.001 respectively. Mean separation within columns by $\mathrm{LSD}$ at $P=0.05$.

Table 3. Effect of AVG rate and various surfactants on fruit firmness, soluble solids, and percent surface red colour of 'Venture' peaches in 2002.

\begin{tabular}{|c|c|c|c|c|c|c|c|c|c|c|c|c|c|c|c|}
\hline \multirow[b]{2}{*}{ Treatment } & \multicolumn{5}{|c|}{ Fruit firmness $(\mathrm{N})$} & \multicolumn{5}{|c|}{ Soluble solids ( ${ }^{\circ}$ Brix) } & \multicolumn{5}{|c|}{ Fruit surface with blush (red) (\%) } \\
\hline & 9 Sept. & 12 Sept. & 16 Sept. & 24 Sept. $^{2}$ & 26 Sept. $^{2}$ & 9 Sept. & 12 Sept. & 16 Sept. & 24 Sept. & 26 Sept. & 9 Sept. & 12 Sept. & 16 Sept. & 24 Sept. & 26 Sept. \\
\hline \multicolumn{16}{|c|}{ Experiment 1-Rate of ReTain ${ }^{y}$} \\
\hline $0 \mathrm{mg} \cdot \mathrm{L}^{-1}$ & 59.7 & 63.9 & --- & 68.5 & 70.8 & 15.2 & 15.1 & --- & 12.4 & 14.5 & 46 & 35 & --- & 27 & 22 \\
\hline $66 \mathrm{mg} \cdot \mathrm{L}^{-1}$ & 75.0 & 74.5 & 64.3 & 86.7 & 79.0 & 13.4 & 14.6 & 15.6 & 12.5 & 14.1 & 53 & 46 & 40 & 36 & 36 \\
\hline $132 \mathrm{mg} \cdot \mathrm{L}^{-1}$ & 81.0 & 76.3 & 74.1 & 88.5 & 86.6 & 14.5 & 14.7 & 14.0 & 14.0 & 15.2 & 58 & 59 & 45 & 44 & 48 \\
\hline $264 \mathrm{mg} \cdot \mathrm{L}^{-1}$ & 86.5 & 94.5 & 73.8 & 94.0 & 94.2 & 15.1 & 15.1 & 16.2 & 12.7 & 16.0 & 57 & 54 & 57 & 46 & 49 \\
\hline Signifigance & $* * *$ & $* * *$ & $* *$ & $* * *$ & $* * *$ & NS & NS & NS & NS & NS & $* *$ & $* * *$ & $* *$ & $* * *$ & $* * *$ \\
\hline $\operatorname{LSD}(p=0.05)$ & 5.9 & 6.0 & 6.1 & 7.4 & 7.1 & 2.46 & 3.72 & 4.40 & 2.52 & 4.71 & 6 & 8 & 8 & 7 & 9 \\
\hline$P$ value & 0.000 & 0.000 & 0.001 & 0.000 & 0.000 & 0.404 & 0.989 & 0.681 & 0.526 & 0.456 & 0.001 & 0.000 & 0.001 & 0.000 & 0.000 \\
\hline \multicolumn{16}{|c|}{ Experiment 2 - Surfactant ${ }^{\mathrm{x}}$} \\
\hline None & 91.8 & 89.2 & 74.3 & 99.0 & 82.1 & 13.8 & 14.5 & 14.7 & 14.0 & 13.5 & 50 & 43 & 49 & 36 & 45 \\
\hline $0.05 \%$ Sylgard 309 & 94.8 & 87.6 & 74.2 & 95.2 & 82.8 & 10.0 & 13.7 & 13.3 & 13.6 & 14.5 & 49 & 46 & 43 & 38 & 44 \\
\hline $0.05 \%$ Regulaid & 88.0 & 81.2 & 69.4 & 92.0 & 82.6 & 12.7 & 14.2 & 14.3 & 13.2 & 13.1 & 37 & 41 & 32 & 35 & 33 \\
\hline $0.50 \%$ LI 700 & 86.5 & 79.5 & 61.4 & 89.6 & 75.5 & 13.4 & 14.5 & 13.6 & 14.0 & 14.7 & 36 & 37 & 39 & 32 & 34 \\
\hline Signifigance & $*$ & $*$ & $*$ & $*$ & NS & NS & NS & NS & NS & NS & $* * *$ & $* * *$ & $*$ & $*$ & $* *$ \\
\hline $\operatorname{LSD}(p=0.05)$ & 6.3 & 7.6 & 8.9 & 7.2 & 7.0 & 3.78 & 3.29 & 5.22 & 4.03 & 4.20 & 6 & 8 & 8 & 7 & 8 \\
\hline$P$ & 0.044 & 0.019 & 0.011 & 0.047 & 0.052 & 0.248 & 0.939 & 0.910 & 0.960 & 0.796 & 0.000 & 0.000 & 0.010 & 0.030 & 0.001 \\
\hline
\end{tabular}

${ }^{2}$ Two weeks after cold storage.

${ }^{y}$ All treatments in Expt. 1 include $0.05 \%$ Sylgard 309 surfactant.

xAll treatments in Expt. 2 include $132 \mathrm{mg} \cdot \mathrm{L}^{-1}$ of Retain.

NS,"***,****Nonsignificant or signifigant differences at $P=0.05,0.01$, or 0.001 respectively. Mean separation within columns by LSD at $P=0.05$. 
penetrometer (fruit texture analyzer model GS14; GÜSS, South Africa) equipped with a 11 $\mathrm{mm}$ probe. Two measurements were made on opposite sides after a about $2 \mathrm{~mm}$ tangential section of skin was peeled from the equator of the fruit. Fruit removed from cold storage were first warmed to $20^{\circ} \mathrm{C}$ before measuring fruit firmness. A composite juice sample from each of the fruit was then collected using a hand juicer and analyzed for percent Brix using a temperature compensating digital refractometer (Atago, Japan).

Table 4. Effect of AVG rate and various surfactants on fruit blush color of 'Venture' peaches in 2002.

\begin{tabular}{|c|c|c|c|c|c|c|c|c|c|c|c|c|c|c|c|}
\hline \multirow[b]{2}{*}{ Treatment } & \multicolumn{5}{|c|}{ Blush color $\mathrm{L}^{*}$} & \multicolumn{5}{|c|}{ Blush color chroma $\left(\mathrm{C}^{*}\right)$} & \multicolumn{5}{|c|}{ Blush color hue angle (Ho) } \\
\hline & 9 Sept. & 12 Sept. & 16 Sept. & 24 Sept. & 26 Sept. & 9 Sept. & 12 Sept. & 16 Sept. & 24 Sept. & 26 Sept. & 9 Sept. & 12 Sept. & 16 Sept. & 24 Sept. & 26 Sept. \\
\hline \multicolumn{16}{|c|}{ Experiment 1 - Rate of ReTain ${ }^{2}$} \\
\hline $0 \mathrm{mg} \cdot \mathrm{L}^{-1}$ & 43.0 & 45.5 & ---- & 47.3 & 47.5 & 29.7 & 30.5 & ---- & 28.1 & 29.7 & 35.7 & 38.9 & ---- & 39.6 & 44.0 \\
\hline $66 \mathrm{mg} \cdot \mathrm{L}^{-1}$ & 42.0 & 41.5 & 43.9 & 44.2 & 43.8 & 26.4 & 28.4 & 27.8 & 25.7 & 25.8 & 31.7 & 30.9 & 34.4 & 34.9 & 33.3 \\
\hline $132 \mathrm{mg} \cdot \mathrm{L}^{-1}$ & 42.2 & 41.3 & 46.0 & 44.0 & 44.5 & 27.5 & 27.0 & 27.5 & 21.9 & 25.0 & 35.9 & 30.9 & 39.6 & 33.8 & 34.4 \\
\hline $264 \mathrm{mg} \cdot \mathrm{L}^{-1}$ & 42.0 & 43.7 & 43.2 & 43.9 & 46.0 & 25.7 & 27.9 & 25.8 & 25.4 & 23.3 & 32.4 & 36.6 & 32.9 & 35.7 & 38.4 \\
\hline Signifigance & NS & $* * *$ & $* *$ & $* * *$ & $*$ & $* *$ & NS & NS & $* * *$ & $*$ & NS & $* *$ & $* *$ & NS & $*$ \\
\hline $\operatorname{LSD}(p=0.05)$ & 1.6 & 1.9 & 1.5 & 1.7 & 2.3 & 2.1 & 2.6 & 2.2 & 2.5 & 2.9 & 4.0 & 4.1 & 3.6 & 4.5 & 5.9 \\
\hline$P$. & 0.541 & 0.000 & 0.001 & 0.000 & 0.048 & 0.002 & 0.127 & 0.108 & 0.000 & 0.010 & 0.074 & 0.000 & 0.001 & 0.056 & 0.017 \\
\hline \multicolumn{16}{|c|}{ Experiment $2-$ Surfactant $^{\mathrm{y}}$} \\
\hline None & 39.8 & 43.6 & 44.1 & 45.8 & 43.2 & 31.4 & 29.9 & 27.2 & 26.8 & 26.6 & 36.1 & 37.1 & 32.9 & 37.7 & 33.2 \\
\hline $0.05 \%$ Sylgard 309 & 39.4 & 44.7 & 47.2 & 46.3 & 44.1 & 27.7 & 30.2 & 32.1 & 26.5 & 27.0 & 33.3 & 41.0 & 42.5 & 40.6 & 36.6 \\
\hline $0.05 \%$ Regulaid & 42.7 & 43.2 & 45.6 & 46.8 & 47.2 & 31.2 & 30.9 & 32.4 & 26.7 & 27.4 & 42.6 & 37.5 & 38.4 & 39.4 & 40.6 \\
\hline $0.50 \%$ LI 700 & 44.6 & 45.5 & 48.0 & 47.4 & 47.0 & 32.5 & 34.3 & 33.0 & 28.7 & 31.8 & 45.0 & 41.4 & 43.7 & 42.5 & 41.9 \\
\hline Signifigance & $* * *$ & NS & $* *$ & NS & $* *$ & $* *$ & $*$ & $* * *$ & NS & NS & $* *$ & NS & $* * *$ & NS & $* * *$ \\
\hline $\operatorname{LSD}(p=0.05)$ & 2.30 & 2.72 & 2.17 & 1.79 & 2.17 & 2.92 & 2.95 & 2.77 & 2.73 & 3.17 & 5.99 & 6.88 & 5.11 & 5.15 & 5.48 \\
\hline$P$ & 0.000 & 0.396 & 0.001 & 0.286 & 0.000 & 0.001 & 0.013 & 0.000 & 0.348 & 0.241 & 0.000 & 0.630 & 0.000 & 0.310 & 0.000 \\
\hline
\end{tabular}

${ }^{\mathrm{z}} \mathrm{All}$ treatments in Expt. 1 include $0.05 \%$ Sylgard 309 surfactant.

${ }^{\mathrm{y}}$ All treatments in Expt. 2 include $132 \mathrm{mg} \cdot \mathrm{L}^{-1}$ of Retain.

NS, $, * *, * * * *$ Nonsignificant or signifigant differences at $P=0.05,0.01$, or 0.001 respectively. Mean separation within columns by LSD at $P=0.05$.

Table 5. Effect of AVG rate and various surfactants on fruit ground color of 'Venture' peaches in 2002.

\begin{tabular}{|c|c|c|c|c|c|c|c|c|c|c|c|c|c|c|c|}
\hline \multirow[b]{2}{*}{ Treatment } & \multicolumn{5}{|c|}{ Ground color $\mathrm{L}^{*}$} & \multicolumn{5}{|c|}{ Ground color chroma $\left(\mathrm{C}^{*}\right)$} & \multicolumn{5}{|c|}{ Ground color hue angle (Ho) } \\
\hline & 9 Sept. & 12 Sept. & 16 Sept. & 24 Sept. & 26 Sept. & 9 Sept. & 12 Sept. & 16 Sept. & 24 Sept. & 26 Sept. & 9 Sept. & 12 Sept. & 16 Sept. & 24 Sept. & 26 Sept. \\
\hline \multicolumn{16}{|c|}{ Experiment 1 - Rate of ReTain ${ }^{2}$} \\
\hline $0 \mathrm{mg} \cdot \mathrm{L}^{-1}$ & 68.3 & 67.2 & ---- & 69.1 & 67.8 & 48.8 & 47.4 & ---- & 46.7 & 47.0 & 225.5 & 167.1 & ---- & 174.2 & 161.5 \\
\hline $66 \mathrm{mg} \cdot \mathrm{L}^{-1}$ & 68.8 & 69.2 & 70.5 & 68.5 & 69.4 & 47.4 & 49.0 & 49.7 & 45.0 & 47.3 & 210.8 & 212.4 & 250.7 & 209.2 & 196.3 \\
\hline $132 \mathrm{mg} \cdot \mathrm{L}^{-1}$ & 67.7 & 68.7 & 69.7 & 68.3 & 69.1 & 46.4 & 47.8 & 47.6 & 42.3 & 44.1 & 209.2 & 252.0 & 228.3 & 234.6 & 214.6 \\
\hline $264 \mathrm{mg} \cdot \mathrm{L}^{-1}$ & 68.1 & 67.8 & 67.7 & 66.7 & 68.5 & 46.4 & 47.0 & 46.6 & 42.2 & 43.8 & 270.4 & 313.7 & 267.5 & 241.7 & 241.5 \\
\hline Signifigance & ns & $* *$ & $* *$ & $* * *$ & ** & $*$ & ns & $*$ & $* * *$ & $* *$ & ns & $* * *$ & ns & ns & $* * *$ \\
\hline $\operatorname{LSD}(p=0.05)$ & 0.8 & 1.2 & 1.3 & 1.0 & 1.2 & 1.7 & 1.9 & 1.6 & 1.8 & 1.9 & 58.0 & 36.7 & 40.4 & 65.3 & 29.5 \\
\hline$P$ & 0.063 & 0.008 & 0.003 & 0.000 & 0.005 & 0.013 & 0.098 & 0.042 & 0.000 & 0.000 & 0.133 & 0.000 & 0.122 & 0.169 & 0.000 \\
\hline \multicolumn{16}{|c|}{ Experiment 2 - Surfactant ${ }^{y}$} \\
\hline None & 67.1 & 67.7 & 69.7 & 68.8 & 68.6 & 46.4 & 47.5 & 47.5 & 45.0 & 46.2 & 204.1 & 258.0 & 209.5 & 195.9 & 211.8 \\
\hline $0.05 \%$ Sylgard 309 & 66.5 & 67.6 & 70.1 & 68.8 & 68.9 & 45.1 & 46.7 & 48.5 & 42.5 & 46.4 & 317.7 & 246.4 & 240.1 & 219.2 & 280.0 \\
\hline $0.05 \%$ Regulaid & 67.3 & 68.1 & 69.6 & 69.0 & 69.5 & 45.7 & 47.7 & 49.1 & 43.6 & 46.4 & 269.8 & 252.4 & 203.5 & 249.3 & 210.9 \\
\hline $0.50 \%$ LI 700 & 67.7 & 68.3 & 69.9 & 69.9 & 69.5 & 46.7 & 48.4 & 49.3 & 45.0 & 47.9 & 232.0 & 221.8 & 193.4 & 230.7 & 199.9 \\
\hline Signifigancez & $\mathrm{ns}$ & $\mathrm{ns}$ & ns & ns & ns & ns & ns & ns & $*$ & ns & $* *$ & $\mathrm{~ns}$ & ns & $\mathrm{ns}$ & $* * *$ \\
\hline $\operatorname{LSD}(p=0.05)$ & 0.9 & 1.1 & 1.2 & 1.1 & 1.0 & 2.2 & 2.1 & 2.3 & 1.9 & 2.1 & 65.4 & 49.2 & 47.9 & 89.0 & 34.8 \\
\hline$P$ & 0.089 & 0.442 & 0.728 & 0.113 & 0.213 & 0.467 & 0.313 & 0.721 & 0.014 & 0.241 & 0.005 & 0.390 & 0.115 & 0.719 & 0.000 \\
\hline
\end{tabular}

${ }^{2}$ All treatments in Expt. 1 include $0.05 \%$ Sylgard 309 surfactant.

${ }^{\mathrm{y}}$ All treatments in Expt. 2 include $132 \mathrm{mg} \cdot \mathrm{L}^{-1}$ of Retain.

${ }_{\text {NS }, *, * * * * * *}$ Nonsignificant or signifigant differences at $P=0.05,0.01$, or 0.001 respectively. Mean separation within columns by LSD at $P=0.05$.

Table 6. Effect of AVG rate and surfactants on yield components and trunk cross-sectional area of 'Babygold 7'and 'Venture' in 2003.

\begin{tabular}{|c|c|c|c|c|c|c|c|}
\hline Treatment & $\begin{array}{c}\text { Surfactant } \\
(\%)\end{array}$ & $\begin{array}{l}\text { Total fruit } \\
\text { harvested } \\
\text { per tree } \\
\text { (no.) }\end{array}$ & $\begin{array}{l}\text { Total yield/tree } \\
\text { (dropped and } \\
\text { harvested fruit) } \\
(\mathrm{kg} / \text { tree })\end{array}$ & $\begin{array}{l}\text { Total yield of } \\
\text { harvested fruit } \\
(\mathrm{kg} / \mathrm{tree})\end{array}$ & $\begin{array}{c}\text { Crop load } \\
\text { (no. fruit } / \mathrm{cm}^{2} \text { ) }\end{array}$ & $\begin{array}{l}\text { Mean fruit wt } \\
\text { of harvest fruit } \\
\text { (g) }\end{array}$ & $\begin{array}{c}\text { Trunk } \\
\text { cross-sectional } \\
\text { area } \\
\left(\mathrm{cm}^{2}\right)\end{array}$ \\
\hline \multicolumn{8}{|l|}{ Babygold 7} \\
\hline $0 \mathrm{mg} \cdot \mathrm{L}^{-1}$ & 0.05 Sylgard 309 & 169 & 28.6 & 23.7 & 6.6 & 140 & 31.4 \\
\hline $66 \mathrm{mg} \cdot \mathrm{L}^{-1}$ & 0.05 Sylgard 309 & 169 & 33.2 & 27.3 & 5.9 & 161 & 36.5 \\
\hline $132 \mathrm{mg} \cdot \mathrm{L}^{-1}$ & 0.05 Sylgard 309 & 157 & 26.9 & 23.0 & 6.3 & 150 & 28.8 \\
\hline $264 \mathrm{mg} \cdot \mathrm{L}^{-1}$ & 0.05 Sylgard 309 & 180 & 31.7 & 29.1 & 6.1 & 164 & 32.8 \\
\hline $132 \mathrm{mg} \cdot \mathrm{L}^{-1}$ & None & 186 & 34.7 & 31.4 & 7.1 & 174 & 31.0 \\
\hline $132 \mathrm{mg} \cdot \mathrm{L}^{-1}$ & 0.05 Regulaid & 181 & 34.5 & 31.1 & 5.5 & 175 & 37.6 \\
\hline $132 \mathrm{mg} \cdot \mathrm{L}^{-1}$ & $0.5 \mathrm{Li}-700$ & 146 & 31.3 & 28.0 & 5.2 & 195 & 32.9 \\
\hline Signifigance & & NS & NS & NS & NS & $*$ & NS \\
\hline $\operatorname{LSD}(p=0.05)$ & & 61 & 11.0 & 9.9 & 2.30 & 31 & 9.4 \\
\hline$P$ & & 0.825 & 0.725 & 0.489 & 0.656 & 0.028 & 0.490 \\
\hline \multicolumn{8}{|l|}{ Venture } \\
\hline $0 \mathrm{mg} \cdot \mathrm{L}^{-1}$ & 0.05 Sylgard 309 & 245 & 46.7 & 40.5 & 5.5 & 166 & 52.3 \\
\hline $66 \mathrm{mg} \cdot \mathrm{L}^{-1}$ & 0.05 Sylgard 309 & 204 & 41.4 & 35.6 & 4.3 & 174 & 57.3 \\
\hline $132 \mathrm{mg} \cdot \mathrm{L}^{-1}$ & 0.05 Sylgard 309 & 223 & 41.4 & 35.8 & 5.1 & 161 & 51.9 \\
\hline $264 \mathrm{mg} \cdot \mathrm{L}^{-1}$ & 0.05 Sylgard 309 & 244 & 49.2 & 42.8 & 5.0 & 176 & 58.0 \\
\hline $132 \mathrm{mg} \cdot \mathrm{L}^{-1}$ & None & 257 & 45.8 & 41.5 & 5.4 & 163 & 54.4 \\
\hline $132 \mathrm{mg} \cdot \mathrm{L}^{-1}$ & 0.05 Regulaid & 227 & 43.0 & 37.7 & 5.8 & 168 & 46.4 \\
\hline $132 \mathrm{mg} \cdot \mathrm{L}^{-1}$ & $0.5 \mathrm{Li}-700$ & 195 & 41.1 & 34.9 & 4.5 & 182 & 53.1 \\
\hline Signifigance & & NS & NS & NS & NS & NS & NS \\
\hline $\operatorname{LSD}(p=0.05)$ & & 74 & 11.4 & 10.6 & 1.4 & 34 & 9.2 \\
\hline$P$ & & 0.569 & 0.662 & 0.590 & 0.277 & 0.856 & 0.217 \\
\hline
\end{tabular}

NS,********N Nonsignificant or signifigant differences at $P=0.05,0.01$, or 0.001 respectively. Mean separation within columns by LSD at $P=0.05$. 
All data were subjected to analysis of variance using SAS PROC GLM procedure (Cary, NC). Mean separation using Protected Fishers Least Significance Difference was used to separate treatment means.

\section{Results and Discussion}

In Expts. 1 and 2, the rate of AVG and type of surfactant had no effect on the total number of fruit harvested per tree, total yield per tree, crop load, or trunk cross-sectional area (Table 1). However, AVG did delay the onset of fruit maturity by about $4 \mathrm{~d}$ (Table 2). This response was similar at all rates of AVG (66 to $246 \mathrm{mg} \cdot \mathrm{L}^{-1}$ ) and independent of the presence of surfactants when AVG was sprayed at a concentration of $132 \mathrm{mg} \cdot \mathrm{L}^{-1}$ (Table 2). For instance, on 12 Sept. all fruit had been harvested from the untreated control trees, while $72 \%$ to $83 \%$ had been harvested from trees treated with AVG and Sylgard 309 surfactant. Furthermore, the rate of AVG or type of surfactant had no effect on fruit drop either preharvest (4 and 6 Sept.) or during the harvest period (Table 2).

AVG had a very positive influence on fruit firmness on 9 Sept. $(P=0.001), 12$ Sept. $(P=$ $0.001), 16$ Sept. $(P=0.01)$, and 2 weeks after air storage at $2{ }^{\circ} \mathrm{C}(P=0.001)$, even though fruit were harvested with similar background colors (Table 3). In general, the firmness response was rate dependent and increased in a curvilinear fashion with increasing rates of AVG. On the first harvest date, there was a $26 \mathrm{~N}$ difference in firmness between fruit treated with 0 and $246 \mathrm{mg} \cdot \mathrm{L}^{-1} \mathrm{AVG}$, and this difference was maintained after 2 weeks in cold storage. Interestingly, fruit firmness appeared to increase after cold storage, perhaps due to slight dehydration during storage. The type of surfactant also had a significant effect on fruit firmness $(P=0.05)$. Trees receiving AVG with either no surfactant or $0.05 \%$ Sylgard 309 had the firmest fruit, while trees receiving AVG with $0.05 \%$ Regulaid or $0.50 \% \mathrm{Li} 700$ had consistently less firm fruit at the three harvest dates and in fruit which were harvested on 12 Sept. and stored for 2 weeks.

Neither rate of AVG nor type of surfactant significantly affected fruit soluble solids at any of the harvest dates or on fruit after cold storage (Table 3 ).

The percentage of surface red color on 9 Sept. $(P=0.01), 12$ Sept. $(P=0.001), 16$ Sept. $(P=0.01)$, and 2 weeks after air storage at $2^{\circ} \mathrm{C}$ was influenced by the rate of $\mathrm{AVG}(P=0.001)$

Table 7. Effect of AVG rate and various surfactants on percent fruit harvested, cumulative fruit harvested, mean fruit weight of 'Venture' and 'Babygold 7' in 2003.

\begin{tabular}{|c|c|c|c|c|c|c|c|c|c|c|c|c|c|c|c|c|c|c|}
\hline \multirow[b]{2}{*}{ ReTain } & \multirow{2}{*}{$\begin{array}{l}\text { Surfactant } \\
(\%)\end{array}$} & \multicolumn{3}{|c|}{$\begin{array}{c}\text { Fruit } \\
\text { harvested } \\
(\% \text { of total } \\
\text { by number })\end{array}$} & \multicolumn{5}{|c|}{$\begin{array}{c}\text { Cumulative } \\
\text { fruit harvested } \\
\text { (\% of total } \\
\text { harvested by no.) }\end{array}$} & \multicolumn{3}{|c|}{$\begin{array}{l}\text { Mean fruit } \\
\text { wt of } \\
\text { harvested } \\
\text { fruit ( } \mathrm{g} / \text { fruit) }\end{array}$} & \multicolumn{6}{|c|}{$\begin{array}{l}\text { Cumulative fruit drop } \\
{[\% \text { dropped of total fruit no. }} \\
\text { (harvested }+ \text { dropped) }]\end{array}$} \\
\hline & & 15 Sept. & $18 \mathrm{Sep}$ & $\overline{\text { Sept }}$ & 25 Sept. & $15 \mathrm{Sep}$ & Sep & 22 Sept. & Sept. & $15 \mathrm{Sept}$ & 18 Sept. & 22 Sept & 25 Sept. & $10 \mathrm{Sept}$ & 15 Sept. & 18 Sept. & $22 \mathrm{Sept}$ & 25 Sept. \\
\hline \multicolumn{19}{|l|}{ Babygold 7} \\
\hline $0 \mathrm{mg} \cdot \mathrm{L}^{-1}$ & 0.05 Sylgard 309 & --- & 36 & 55 & 9 & 0 & 36 & 91 & 100 & --- & 141 & 148 & 115 & 1.6 & 4.2 & 7.6 & 14.6 & 15.9 \\
\hline $66 \mathrm{mg} \cdot \mathrm{L}^{-1}$ & 0.05 Sylgard 309 & - & 21 & 43 & 36 & 0 & 21 & 64 & 100 & --- & 182 & 163 & 147 & 2.3 & 4.3 & 6.7 & 12.1 & 18.1 \\
\hline $132 \mathrm{mg} \cdot \mathrm{L}^{-1}$ & 0.05 Sylgard 309 & _- & 25 & 32 & 43 & 0 & 25 & 57 & 100 & --- & 160 & 155 & 143 & 2.1 & 2.5 & 3.7 & 10.2 & 16.3 \\
\hline $264 \mathrm{mg} \cdot \mathrm{L}^{-1}$ & 0.05 Sylgard 309 & --- & 20 & 31 & 49 & 0 & 20 & 51 & 100 & --- & 177 & 178 & 155 & 1.2 & 2.1 & 4.6 & 6.5 & 8.5 \\
\hline $132 \mathrm{mg} \cdot \mathrm{L}^{-1}$ & None & - & 19 & 34 & 47 & 0 & 19 & 53 & 100 & --- & 180 & 183 & 161 & 1.6 & 3.0 & 5.3 & 8.1 & 11.5 \\
\hline $132 \mathrm{mg} \cdot \mathrm{L}^{-1}$ & 0.05 Regulaid & --- & 19 & 34 & 47 & 0 & 19 & 53 & 100 & --- & 189 & 199 & 160 & 1.3 & 2.3 & 4.2 & 6.3 & 10.8 \\
\hline $132 \mathrm{mg} \cdot \mathrm{L}^{-1}$ & $0.5 \mathrm{Li}-700$ & --- & 26 & 34 & 40 & 0 & 26 & 60 & 100 & --- & 174 & 279 & 162 & 2.2 & 3.9 & 7.6 & 10.7 & 15.4 \\
\hline Signifigance & & --- & NS & $* *$ & $* *$ & NS & NS & $* *$ & --- & --- & NS & NS & NS & NS & NS & NS & NS & NS \\
\hline $\operatorname{LSD}(p=0.0$ & 05) & --- & 15.7 & 20.6 & 20.6 & --- & 15.7 & 20.6 & --- & --- & 37.03 & 85.63 & 26.16 & 1.92 & 2.82 & 4.00 & 6.03 & 7.89 \\
\hline$P$ & & --- & 0.263 & 0.007 & 0.007 & --- & 0.263 & 0.007 & --- & --- & 0.179 & 0.068 & 0.088 & 0.823 & 0.479 & 0.250 & 0.081 & 0.161 \\
\hline \multicolumn{19}{|l|}{ Venture } \\
\hline $0 \mathrm{mg} \cdot \mathrm{L}^{-1}$ & 0.05 Sylgard 309 & 33 & 15 & 33 & 19 & 33 & 48 & 82 & 100 & 180 & 180 & 157 & 133 & 2.0 & 4.9 & 6.5 & 12.7 & 16.3 \\
\hline $66 \mathrm{mg} \cdot \mathrm{L}^{-1}$ & 0.05 Sylgard 309 & 17 & 12 & 25 & 46 & 17 & 29 & 54 & 100 & 187 & 182 & 181 & 163 & 2.2 & 3.0 & 4.3 & 6.9 & 16.2 \\
\hline $132 \mathrm{mg} \cdot \mathrm{L}^{-1}$ & 0.05 Sylgard 309 & 12 & 9 & 38 & 41 & 12 & 21 & 59 & 100 & 182 & 171 & 165 & 151 & 1.5 & 2.2 & 3.0 & 8.2 & 15.3 \\
\hline $264 \mathrm{mg} \cdot \mathrm{L}^{-1}$ & 0.05 Sylgard 309 & 11 & 6 & 18 & 65 & 11 & 17 & 35 & 100 & 191 & 187 & 204 & 166 & 2.5 & 4.3 & 4.8 & 7.0 & 15.2 \\
\hline $132 \mathrm{mg} \cdot \mathrm{L}^{-1}$ & None & 15 & 9 & 34 & 43 & 15 & 24 & 57 & 100 & 181 & 169 & 173 & 150 & 2.7 & 3.8 & 4.3 & 6.7 & 11.8 \\
\hline $132 \mathrm{mg} \cdot \mathrm{L}^{-1}$ & 0.05 Regulaid & 21 & 10 & 39 & 30 & 21 & 32 & 71 & 100 & 175 & 170 & 171 & 159 & 2.4 & 2.7 & 3.3 & 7.7 & 15.5 \\
\hline $132 \mathrm{mg} \cdot \mathrm{L}^{-1}$ & $0.5 \mathrm{Li}-700$ & 19 & 12 & 27 & 42 & 19 & 31 & 58 & 100 & 196 & 184 & 197 & 164 & 3.1 & 4.9 & 6.0 & 10.3 & 17.2 \\
\hline Signifigance & & NS & NS & NS & NS & NS & NS & $*$ & --- & NS & NS & NS & NS & NS & NS & NS & NS & NS \\
\hline $\operatorname{LSD}(p=0.0$ & & 13.9 & 19.3 & 25.9 & 25.9 & 13.9 & 19.3 & 25.5 & --- & 38.42 & 33.73 & 34.99 & 34.01 & 2.97 & 4.12 & 4.96 & 5.46 & 7.99 \\
\hline$P$ & & 0.054 & 0.407 & 0.107 & 0.107 & 0.054 & 0.065 & 0.042 & --- & 0.925 & 0.845 & 0.104 & 0.546 & 0.950 & 0.709 & 0.742 & 0.248 & 0.870 \\
\hline
\end{tabular}

Nonsignificant or signifigant differences at $P=0.05,0.01$, or 0.001 respectively. Mean separation within columns by LSD at $P=0.05$

Table 8. Effect of AVG rate and various surfactants on fruit firmness of 'Venture' and 'Babygold 7' in 2003.

\begin{tabular}{|c|c|c|c|c|c|c|c|}
\hline \multirow[b]{3}{*}{ Treatment } & \multirow{3}{*}{$\begin{array}{c}\text { Surfactant } \\
(\%)\end{array}$} & \multicolumn{6}{|c|}{ Firmness (N) } \\
\hline & & \multirow[b]{2}{*}{15 Sept. } & \multirow[b]{2}{*}{19 Sept. } & \multirow[b]{2}{*}{22 Sept. } & \multirow[b]{2}{*}{25 Sept. } & \multicolumn{2}{|c|}{ After storage } \\
\hline & & & & & & 6 Oct. & 10 Oct. \\
\hline \multicolumn{8}{|l|}{ Babygold 7} \\
\hline $0 \mathrm{mg} \cdot \mathrm{L}^{-1}$ & 0.05 Sylgard 309 & --- & 56.1 & 46.7 & 40.2 & 52.3 & 38.8 \\
\hline $66 \mathrm{mg} \cdot \mathrm{L}^{-1}$ & 0.05 Sylgard 309 & --- & 65.0 & 58.2 & 47.5 & 61.1 & 46.7 \\
\hline $132 \mathrm{mg} \cdot \mathrm{L}^{-1}$ & 0.05 Sylgard 309 & --- & 69.3 & 60.4 & 52.8 & 69.3 & 51.9 \\
\hline $264 \mathrm{mg} \cdot \mathrm{L}^{-1}$ & 0.05 Sylgard 309 & --- & 78.7 & 65.0 & 64.8 & 74.9 & 61.0 \\
\hline $132 \mathrm{mg} \cdot \mathrm{L}^{-1}$ & None & --- & 72.1 & 61.2 & 54.2 & 69.5 & 51.0 \\
\hline $132 \mathrm{mg} \cdot \mathrm{L}^{-1}$ & 0.05 Regulaid & --- & 73.7 & 61.5 & 51.4 & 70.9 & 51.1 \\
\hline $132 \mathrm{mg} \cdot \mathrm{L}^{-1}$ & $0.5 \mathrm{Li}-700$ & - & 77.5 & 63.6 & 57.3 & 75.1 & 52.6 \\
\hline Signifigance & & --- & $* * *$ & $* * *$ & $* * *$ & $* * *$ & $* * *$ \\
\hline $\operatorname{LSD}(p=0.05)$ & & --- & 3.5 & 3.0 & 3.6 & 3.6 & 3.3 \\
\hline$P$ & & --- & $<0.0001$ & $<0.0001$ & $<0.0001$ & $<0.0001$ & $<0.0001$ \\
\hline \multicolumn{8}{|l|}{ Venture } \\
\hline $0 \mathrm{mg} \cdot \mathrm{L}^{-1}$ & 0.05 Sylgard 309 & 58.1 & 57.7 & 48.7 & 46.2 & 56.2 & 48.8 \\
\hline $66 \mathrm{mg} \cdot \mathrm{L}^{-1}$ & 0.05 Sylgard 309 & 70.3 & 70.7 & 63.7 & 58.0 & 73.9 & 58.5 \\
\hline $132 \mathrm{mg} \cdot \mathrm{L}^{-1}$ & 0.05 Sylgard 309 & 70.7 & 69.1 & 64.0 & 56.3 & 72.1 & 59.8 \\
\hline $264 \mathrm{mg} \cdot \mathrm{L}^{-1}$ & 0.05 Sylgard 309 & 83.0 & 82.3 & 73.3 & 65.0 & 80.6 & 62.7 \\
\hline $132 \mathrm{mg} \cdot \mathrm{L}^{-1}$ & None & 64.9 & 67.6 & 55.5 & 49.4 & 56.6 & 49.1 \\
\hline $132 \mathrm{mg} \cdot \mathrm{L}^{-1}$ & 0.05 Regulaid & 66.5 & 68.5 & 56.8 & 48.6 & 68.4 & 50.5 \\
\hline $132 \mathrm{mg} \cdot \mathrm{L}^{-1}$ & $0.5 \mathrm{Li}-700$ & 67.6 & 72.7 & 62.5 & 51.7 & 68.0 & 51.0 \\
\hline Signifigance & & $* * *$ & $* * *$ & $* * *$ & $* * *$ & $* * *$ & $* * *$ \\
\hline $\operatorname{LSD}(p=0.05)$ & & 4.7 & 4.0 & 3.6 & 3.9 & 5.2 & 4.2 \\
\hline$P$ & & $<0.0001$ & $<0.0001$ & $<0.0001$ & $<0.0001$ & $<0.0001$ & $<0.0001$ \\
\hline
\end{tabular}

NS,********N Nonsignificant or signifigant differences at $P=0.05,0.01$, or 0.001 respectively. Mean separation within columns by LSD at $P=0.05$. 
(Table 3). Percentage of surface red increased with increasing rates of AVG. Trees receiving AVG with either no surfactant or $0.05 \%$ Sylgard 309 had more surface red color than fruit from trees receiving AVG with $0.05 \%$ Regulaid or $0.50 \%$ Li 700 .

Fruit blush color L*, chroma and hue angle values were generally lower on fruit treated with AVG, regardless of the rate of AVG applied (Table 4). Also, trees treated with Regulaid and Li 700 had higher blush L*, chroma and hue angle values. Likewise, there was a significant rate effect on fruit ground color L*, chroma and hue angle values; when this effect was significant at a particular harvest date, the $\mathrm{L}^{*}$ and chroma values were lower, and hue angle values were higher, at higher rates of AVG.
The influence of surfactants on fruit ground color L*, chroma and hue angle values were inconsistent (Table 5).

In Expts. 3 and 4, the rate of AVG and type surfactant had no significant effect on the total number of fruit harvested per tree, total yield per tree, crop load, or trunk cross-sectional area for both 'Babygold 7' and 'Venture' (Table 6). 'Babygold 7' fruit treated with AVG appeared to have greater mean fruit weight, however, when fruit weight was analysed with crop load as a co-variate, this effect was not significant, indicating that fruit size was influenced more by crop load than AVG treatment. AVG did however significantly delay the fruit maturity for 'Babygold 7' and 'Venture' by about 3 and $4 \mathrm{~d}$, respectively (Table 7). There was no significant AVG or surfactant effect on mean fruit weight or fruit drop at any of the harvest dates for either cultivar (Table 7).

As was found in Expts. 1 and 2, AVG had a significant effect on fruit firmness during harvest (Fig. 1) and after storage (Table 8) for both 'Venture' and 'Babygold 7'. Firmness increased in a quadratic fashion with increasing rates of AVG at all harvest dates, with the exception of 'Babygold 7' harvest on 25 Sept., which increased in a linear fashion (Fig 1a). The effect of surfactants was inconsistent and much less dramatic than the rate effect of AVG (Table 8). 'Babygold 7' and 'Venture' trees treated with $0.05 \% \mathrm{Li}-700$, generally had fruit with greater firmness than trees treated with either $0.05 \%$ Regulaid or no surfactant, when compared at

Table 9. Effect of AVG rate and various surfactants on fruit blush color of 'Babygold 7' and 'Venture' peaches in 2003.

\begin{tabular}{|c|c|c|c|c|c|c|c|c|c|c|c|c|c|c|c|c|c|c|c|c|c|}
\hline \multirow[b]{4}{*}{ Treatment } & \multirow[b]{4}{*}{$\begin{array}{c}\text { Surfactant } \\
(\%)\end{array}$} & \multicolumn{20}{|c|}{ Blush color } \\
\hline & & \multicolumn{6}{|c|}{$\mathrm{L}^{*}$} & \multicolumn{7}{|c|}{ Chroma $\left(C^{*}\right)$} & \multicolumn{7}{|c|}{ Hue angle (Ho) } \\
\hline & & \multicolumn{6}{|c|}{ After storage } & \multicolumn{7}{|c|}{ After storage } & \multicolumn{7}{|c|}{ After storage } \\
\hline & & $\begin{array}{c}15 \\
\text { Sept. }\end{array}$ & $\begin{array}{c}19 \\
\text { Sept. }\end{array}$ & $\begin{array}{c}22 \\
\text { Sept. }\end{array}$ & $\begin{array}{c}25 \\
\text { Sept. }\end{array}$ & $\begin{array}{c}6 \\
\text { Oct. }\end{array}$ & $\begin{array}{c}10 \\
\text { Oct. }\end{array}$ & $\begin{array}{c}15 \\
\text { Sept. }\end{array}$ & $\begin{array}{c}19 \\
\text { Sept. }\end{array}$ & $\begin{array}{c}22 \\
\text { Sept. }\end{array}$ & $\begin{array}{c}25 \\
\text { Sept. }\end{array}$ & $\begin{array}{c}6 \\
\text { Oct. }\end{array}$ & $\begin{array}{c}10 \\
\text { Oct. }\end{array}$ & $\begin{array}{c}14 \\
\text { Oct. }\end{array}$ & $\begin{array}{c}15 \\
\text { Sept. }\end{array}$ & $\begin{array}{c}19 \\
\text { Sept. }\end{array}$ & $\begin{array}{c}22 \\
\text { Sept. }\end{array}$ & $\begin{array}{c}25 \\
\text { Sept. }\end{array}$ & $\begin{array}{c}6 \\
\text { Oct. }\end{array}$ & $\begin{array}{c}10 \\
\text { Oct. }\end{array}$ & $\begin{array}{c}14 \\
\text { Oct. }\end{array}$ \\
\hline \multicolumn{22}{|l|}{ Babygold 7} \\
\hline $0 \mathrm{mg} \cdot \mathrm{L}^{-1}$ & 0.05 Sylgard 309 & -- & 48.2 & 50.3 & 51.7 & 47.6 & 49.9 & -- & 28.8 & 31.2 & 35.5 & 28.4 & 30.3 & 35.8 & -- & 35.1 & 41.8 & 45.4 & 34.4 & 38.9 & 47.9 \\
\hline $66 \mathrm{mg} \cdot \mathrm{L}^{-1}$ & 0.05 Sylgard 309 & --- & 48.6 & 49.7 & 50.2 & 48.0 & 47.8 & --- & 29.7 & 32.8 & 33.1 & 29.4 & 29.8 & 33.9 & --- & 36.7 & 41.7 & 43.2 & 35.9 & 35.7 & 46.2 \\
\hline $132 \mathrm{mg} \cdot \mathrm{L}^{-1}$ & 0.05 Sylgard 309 & --- & 45.3 & 46.5 & 46.7 & 46.6 & 47.4 & --- & 26.7 & 29.6 & 30.4 & 28.6 & 28.1 & 33.2 & --- & 29.5 & 34.0 & 36.5 & 32.9 & 34.2 & 44.2 \\
\hline $264 \mathrm{mg} \cdot \mathrm{L}^{-1}$ & 0.05 Sylgard 309 & --- & 45.0 & 45.6 & 43.3 & 44.6 & 43.5 & --- & 25.1 & 26.7 & 26.3 & 25.7 & 25.6 & 28.9 & --- & 29.8 & 30.9 & 28.1 & 28.5 & 28.3 & 36.3 \\
\hline $132 \mathrm{mg} \cdot \mathrm{L}^{-1}$ & None & -- & 45.3 & 44.1 & 45.1 & 46.0 & 45.5 & -- & 26.8 & 26.4 & 29.1 & 27.7 & 26.6 & 28.0 & -- & 29.8 & 29.0 & 31.6 & 31.5 & 31.5 & 31.9 \\
\hline $132 \mathrm{mg} \cdot \mathrm{L}^{-1}$ & 0.05 Regulaid & --- & 47.8 & 47.9 & 48.6 & 45.6 & 47.1 & --- & 28.2 & 30.5 & 31.1 & 27.3 & 28.3 & 32.7 & --- & 35.0 & 35.3 & 37.5 & 31.5 & 34.0 & 42.9 \\
\hline $132 \mathrm{mg} \cdot \mathrm{L}^{-1}$ & $0.5 \mathrm{Li}-700$ & -- & 46.6 & 46.0 & 45.7 & 46.4 & 45.9 & --- & 28.1 & 28.1 & 28.7 & 27.7 & 26.5 & 30.4 & -- & 31.8 & 32.3 & 32.4 & 31.8 & 31.5 & 39.8 \\
\hline Signifigance & & -- & $* * *$ & $* * *$ & $* * *$ & $*$ & $* * *$ & --- & $*$ & $* * *$ & $* * *$ & NS & $* * *$ & $* * *$ & --- & $* * *$ & $* * *$ & $* * *$ & $*$ & $* * *$ & $* * *$ \\
\hline $\operatorname{LSD}(p=0.0$ & 05) & -- & 1.83 & 1.88 & 1.95 & 1.80 & 1.81 & -- & 2.23 & 1.86 & 2.05 & 1.96 & 2.08 & 2.86 & -- & 3.56 & 3.67 & 3.74 & 3.29 & 3.24 & 5.23 \\
\hline$P$ & & -- & 0.000 & $<0.0001$ & $<0.0001$ & 0.017 & $<0.0001$ & --- & 0.018 & $<0.0001$ & $<0.0001$ & 0.067 & $<0.0001<$ & $<0.0001$ & -- & $<0.0001$ & $<0.0001$ & $<0.0001$ & 0.004 & $<0.0001$ & $<0.0001$ \\
\hline \multicolumn{22}{|c|}{$0.018<0.0001<0.0001<0.00 /<0.0001<0.0001 \quad---<0.0001<0.0001<0.0001 \quad 0.004<0.0001<0.0001$} \\
\hline $0 \mathrm{mg} \cdot \mathrm{L}^{-1}$ & 0.05 Sylgard 309 & 43.0 & 49.5 & 48.1 & 52.6 & 46.9 & 46.3 & 27.6 & 29.3 & 30.8 & 35.8 & 29.4 & 27.0 & -- & 29.5 & 39.3 & 38.1 & 47.5 & 33.5 & 32.8 & -- \\
\hline $66 \mathrm{mg} \cdot \mathrm{L}^{-1}$ & 0.05 Sylgard 309 & 43.0 & 52.3 & 47.4 & 49.6 & 45.2 & 48.6 & 25.5 & 30.8 & 29.5 & 32.5 & 25.8 & 29.3 & -- & 29.7 & 48.0 & 35.8 & 42.9 & 31.3 & 37.5 & -- \\
\hline $132 \mathrm{mg} \cdot \mathrm{L}^{-1}$ & 0.05 Sylgard 309 & 42.4 & 48.7 & 50.1 & 51.8 & 46.8 & 46.4 & 27.3 & 29.7 & 32.9 & 35.3 & 29.2 & 30.8 & -- & 28.8 & 36.3 & 41.2 & 48.6 & 34.8 & 35.1 & -- \\
\hline $264 \mathrm{mg} \cdot \mathrm{L}^{-1}$ & 0.05 Sylgard 309 & 43.4 & 45.2 & 49.8 & 50.3 & 45.5 & 46.1 & 26.1 & 23.5 & 29.7 & 30.8 & 26.1 & 25.6 & - & 30.1 & 30.6 & 40.0 & 44.2 & 31.5 & 33.4 & -- \\
\hline $132 \mathrm{mg} \cdot \mathrm{L}^{-1}$ & None & 42.9 & 47.6 & 50.4 & 52.9 & 45.8 & 45.6 & 25.6 & 26.3 & 32.2 & 35.8 & 25.3 & 27.8 & -- & 29.6 & 34.8 & 41.7 & 49.2 & 32.3 & 32.1 & --- \\
\hline $132 \mathrm{mg} \cdot \mathrm{L}^{-1}$ & 0.05 Regulaid & 43.3 & 45.8 & 53.4 & 54.5 & 45.2 & 48.3 & 26.0 & 26.2 & 35.6 & 37.7 & 28.2 & 31.1 & -- & 28.5 & 31.4 & 47.8 & 51.6 & 30.7 & 36.9 & -- \\
\hline $132 \mathrm{mg} \cdot \mathrm{L}^{-1}$ & $0.5 \mathrm{Li}-700$ & 42.6 & 46.9 & 50.1 & 49.2 & 45.1 & 45.8 & 25.7 & 25.9 & 31.3 & 32.2 & 25.9 & 28.2 & -- & 28.6 & 33.7 & 41.3 & 42.3 & 31.0 & 32.2 & -- \\
\hline Signifigance & & NS & $* * *$ & $*$ & NS & NS & $*$ & NS & $* * *$ & $* *$ & $* *$ & NS & $* * *$ & -- & NS & $* * *$ & $*$ & NS & NS & $*$ & -- \\
\hline $\operatorname{LSD}(p=0.0$ & 05) & 1.65 & 2.50 & 3.27 & 3.88 & 2.63 & 2.11 & 2.30 & 3.22 & 3.07 & 3.62 & 3.17 & 2.79 & -- & 3.04 & 5.11 & 6.66 & 7.54 & 4.53 & 3.70 & -- \\
\hline$P$ & & 0.902 & $<0.0001$ & 10.014 & 0.124 & 0.690 & 0.021 & 0.428 & $<0.0001$ & 0.002 & 0.006 & 0.183 & 0.001 & -- & 0.916 & $<0.0001$ & 0.026 & 0.153 & 0.581 & 0.011 & --- \\
\hline
\end{tabular}

Table 10. Effect of AVG rate and various surfactants on fruit ground color of 'Babygold 7' and 'Venture' peaches in 2003

\begin{tabular}{|c|c|c|c|c|c|c|c|c|c|c|c|c|c|c|c|c|c|c|c|c|c|}
\hline \multirow[b]{4}{*}{ Treatment } & \multirow[b]{4}{*}{$\begin{array}{c}\text { Surfactant } \\
(\%)\end{array}$} & \multicolumn{20}{|c|}{ Blush color } \\
\hline & & \multicolumn{6}{|c|}{$\mathrm{L}^{*}$} & \multicolumn{7}{|c|}{ Chroma (C*) } & \multicolumn{7}{|c|}{ Hue angle (Ho) } \\
\hline & & \multicolumn{6}{|c|}{ After storage } & \multicolumn{7}{|c|}{ After storage } & \multicolumn{7}{|c|}{ After storage } \\
\hline & & $\begin{array}{c}15 \\
\text { Sept. }\end{array}$ & $\begin{array}{c}19 \\
\text { Sept. }\end{array}$ & $\begin{array}{c}22 \\
\text { Sept. }\end{array}$ & $\begin{array}{c}25 \\
\text { Sept. }\end{array}$ & $\begin{array}{c}6 \\
\text { Oct. }\end{array}$ & $\begin{array}{c}10 \\
\text { Oct. }\end{array}$ & $\begin{array}{c}15 \\
\text { Sept. }\end{array}$ & $\begin{array}{c}19 \\
\text { Sept. }\end{array}$ & $\begin{array}{c}22 \\
\text { Sept. }\end{array}$ & $\begin{array}{c}25 \\
\text { Sept. }\end{array}$ & $\begin{array}{c}6 \\
\text { Oct. }\end{array}$ & $\begin{array}{l}10 \\
\text { Oct. }\end{array}$ & $\begin{array}{c}14 \\
\text { Oct. }\end{array}$ & $\begin{array}{c}15 \\
\text { Sept. }\end{array}$ & $\begin{array}{c}19 \\
\text { Sept. }\end{array}$ & $\begin{array}{c}22 \\
\text { Sept. }\end{array}$ & $\begin{array}{c}25 \\
\text { Sept. }\end{array}$ & $\begin{array}{c}6 \\
\text { Oct. }\end{array}$ & $\begin{array}{l}10 \\
\text { Oct. }\end{array}$ & $\begin{array}{c}14 \\
\text { Oct. }\end{array}$ \\
\hline \multicolumn{22}{|l|}{ Babygold 7} \\
\hline $0 \mathrm{mg} \cdot \mathrm{L}^{-1}$ & 0.05 Sylgard 309 & --- & 71.3 & 67.8 & 70.6 & 70.7 & 71.6 & --- & 45.8 & 42.2 & 46.4 & 43.1 & 42.9 & 44.1 & --- & 84.4 & 76.9 & 85.2 & 79.9 & 80.4 & 79.8 \\
\hline $66 \mathrm{mg} \cdot \mathrm{L}^{-1}$ & 0.05 Sylgard 309 & --- & 71.2 & 69.9 & 70.5 & 72.3 & 71.5 & --- & 44.2 & 45.1 & 45.5 & 43.8 & 44.7 & 44.8 & --- & 86.8 & 86.2 & 85.8 & 83.9 & 82.5 & 83.5 \\
\hline $132 \mathrm{mg} \cdot \mathrm{L}^{-1}$ & 0.05 Sylgard 309 & --- & 71.0 & 70.6 & 69.2 & 71.3 & 71.3 & --- & 45.8 & 45.0 & 44.7 & 44.1 & 43.9 & 43.7 & --- & 84.6 & 84.6 & 84.4 & 84.3 & 84.2 & 79.7 \\
\hline $264 \mathrm{mg} \cdot \mathrm{L}^{-1}$ & 0.05 Sylgard 309 & --- & 70.7 & 70.0 & 69.8 & 70.8 & 71.1 & --- & 44.0 & 42.6 & 43.4 & 42.8 & 43.1 & 42.7 & --- & 87.2 & 84.0 & 87.6 & 83.6 & 84.8 & 83.3 \\
\hline $132 \mathrm{mg} \cdot \mathrm{L}^{-1}$ & None & --- & 70.6 & 68.6 & 68.6 & 70.5 & 70.0 & --- & 42.4 & 42.5 & 43.1 & 42.1 & 42.2 & 41.5 & --- & 87.5 & 81.5 & 83.3 & 83.5 & 81.8 & 77.9 \\
\hline $132 \mathrm{mg} \cdot \mathrm{L}^{-1}$ & 0.05 Regulaid & --- & 71.0 & 70.9 & 70.1 & 70.8 & 70.7 & -- & 43.8 & 43.4 & 44.9 & 42.2 & 43.0 & 42.6 & --- & 87.4 & 85.0 & 85.3 & 83.0 & 82.2 & 80.6 \\
\hline $132 \mathrm{mg} \cdot \mathrm{L}^{-1}$ & $0.5 \mathrm{Li}-700$ & --- & 70.8 & 70.5 & 70.5 & 71.4 & 71.4 & --- & 43.4 & 43.8 & 44.2 & 42.4 & 42.5 & 41.4 & -- & 90.3 & 85.9 & 87.7 & 84.8 & 84.4 & 80.8 \\
\hline Signifigance & & --- & NS & NS & $*$ & NS & $*$ & --- & $* * *$ & $* * *$ & $* * *$ & $* *$ & $* * *$ & $* * *$ & -- & $* *$ & $* * *$ & $* * *$ & $*$ & $* * *$ & $* * *$ \\
\hline $\operatorname{LSD}(p=0.05$ & & --- & 1.04 & 1.82 & 1.16 & 1.28 & 0.92 & --- & 1.62 & 1.57 & 1.44 & 1.24 & 1.19 & 1.62 & -- & 2.77 & 3.27 & 2.36 & 2.90 & 2.08 & 3.30 \\
\hline$P$ & & --- & 0.882 & 0.005 & 0.002 & 0.078 & 0.012 & --- & 0.000 & $<0.0001$ & 0.000 & 0.003 & $0.000<$ & $<0.0001$ & -- & 0.001 & $<0.0001$ & 0.001 & 0.020 & $<0.0001$ & 0.000 \\
\hline \multicolumn{22}{|c|}{ ( } \\
\hline $0 \mathrm{mg} \cdot \mathrm{L}^{-1}$ & 0.05 Sylgard 309 & 70.4 & 69.6 & 70.4 & 69.8 & 71.5 & 70.4 & 47.1 & 44.8 & 46.7 & 49.0 & 45.6 & 46.3 & --- & 81.8 & 80.1 & 81.9 & 80.8 & 80.6 & 78.2 & --- \\
\hline $66 \mathrm{mg} \cdot \mathrm{L}^{-1}$ & 0.05 Sylgard 309 & 70.2 & 70.1 & 71.6 & 69.7 & 71.6 & 70.8 & 45.8 & 44.5 & 46.1 & 47.3 & 44.7 & 45.7 & -- & 85.3 & 84.7 & 85.9 & 83.3 & 83.6 & 81.3 & --- \\
\hline $132 \mathrm{mg} \cdot \mathrm{L}^{-1}$ & 0.05 Sylgard 309 & 70.4 & 71.3 & 70.9 & 69.6 & 77.2 & 70.6 & 46.3 & 45.2 & 51.3 & 47.2 & 46.9 & 47.0 & -- & 85.3 & 85.0 & 85.2 & 84.8 & 81.1 & 79.8 & --- \\
\hline $264 \mathrm{mg} \cdot \mathrm{L}^{-1}$ & 0.05 Sylgard 309 & 70.5 & 71.0 & 70.6 & 68.1 & 71.9 & 71.2 & 43.5 & 42.9 & 44.1 & 44.0 & 44.4 & 43.8 & --- & 86.7 & 88.8 & 87.5 & 84.4 & 86.6 & 84.7 & --- \\
\hline $132 \mathrm{mg} \cdot \mathrm{L}^{-1}$ & None & 68.2 & 70.7 & 70.8 & 68.5 & 71.0 & 70.8 & 42.1 & 43.0 & 45.6 & 46.3 & 43.2 & 44.0 & --- & 82.2 & 86.4 & 84.4 & 81.6 & 83.1 & 79.7 & -- \\
\hline $132 \mathrm{mg} \cdot \mathrm{L}^{-1}$ & 0.05 Regulaid & 70.7 & 71.5 & 70.3 & 67.8 & 71.2 & 71.0 & 45.2 & 45.3 & 45.6 & 46.9 & 45.2 & 45.6 & --- & 83.9 & 84.6 & 81.4 & 78.7 & 81.5 & 80.4 & --- \\
\hline $132 \mathrm{mg} \cdot \mathrm{L}^{-1}$ & $0.5 \mathrm{Li}-700$ & 69.2 & 70.5 & 70.5 & 69.2 & 70.5 & 71.2 & 43.9 & 43.2 & 45.3 & 47.6 & 42.9 & 44.8 & --- & 82.8 & 88.1 & 83.7 & 83.3 & 82.8 & 80.6 & --- \\
\hline Signifigance & & $* * *$ & $*$ & NS & NS & NS & NS & $* * *$ & $*$ & $*$ & $* * *$ & $* *$ & $* * *$ & --- & $* *$ & $* * *$ & $* *$ & $* *$ & NS & $* * *$ & --- \\
\hline $\operatorname{LSD}(p=0.05$ & & 1.19 & 1.18 & 1.26 & 1.48 & 6.56 & 0.92 & 1.44 & 1.76 & 4.31 & 1.57 & 1.78 & 1.35 & --- & 2.64 & 2.63 & 3.49 & 3.36 & 3.78 & 1.99 & -- \\
\hline$P$ & & 0.000 & 0.015 & 0.421 & 0.062 & 0.509 & 0.468 & $<0.0001$ & 0.014 & 0.043 & $<0.0001$ & 0.009 & $<0.0001$ & -- & 0.001 & $<0.0001$ & 10.008 & 0.006 & 0.271 & $<0.0001$ & --- \\
\hline
\end{tabular}


the same rate of $132 \mathrm{mg} \cdot \mathrm{L}^{-1} \mathrm{AVG}$. This effect was observed for all the harvest dates, although not always statistically significant.

AVG reduced while surfactants generally increased blush $\mathrm{L}^{*}$, chroma and hue angle values (Tables 9). For AVG, this effect was rate dependent and inversely related to concentration; fruit treated with the highest rates of AVG had the lowest $\mathrm{L}^{*}$, chroma and hue angle values. The effect of surfactants was less dramatic and less consistent.

AVG and the surfactants had a more variable

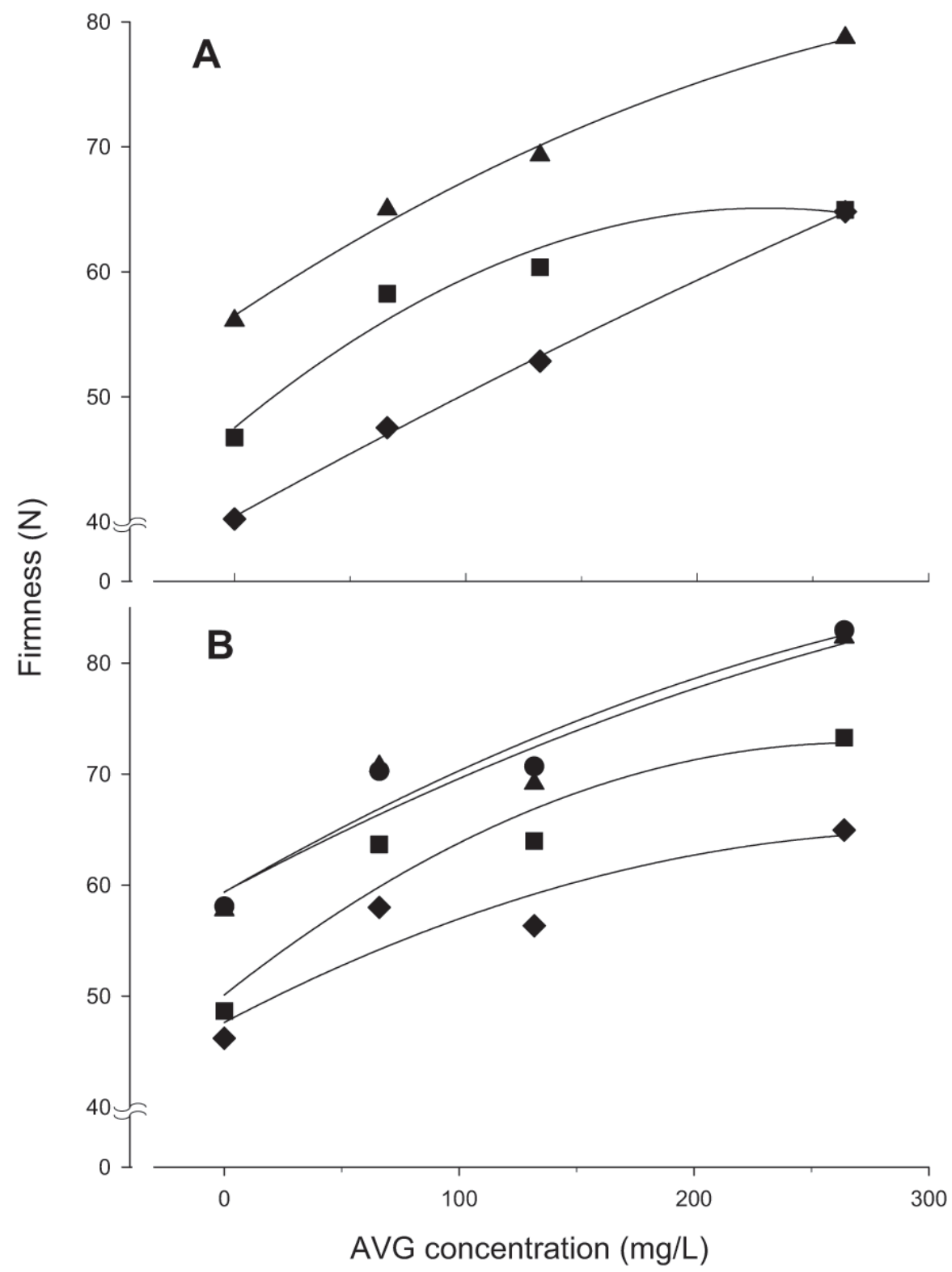

Fig. 1. Relationship between AVG concentration and fruit firmness for 'Babygold 7' (A) and 'Venture' (B)

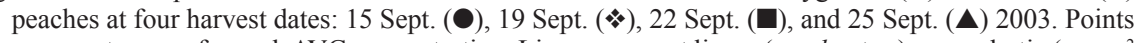
represent means for each AVG concentration. Lines represent linear $(y=b x+\mathrm{c})$ or quadratic $\left(y=\mathrm{a} x^{2}\right.$ $+\mathrm{b} x+c$ ) regression equations, where $x=\mathrm{AVG}$ concentration. Specific coefficients are:

\begin{tabular}{lccccc} 
Cultivar & Harvest date & $\mathrm{a}$ & $\mathrm{b}$ & $\mathrm{c}$ & $r$ \\
\hline Babygold 7 & 19 Sept. & --- & 0.10 & 40.42 & 0.999 \\
& 22 Sept. & -0.0003 & 0.15 & 47.52 & 0.979 \\
Venture & 25 Sept & -0.0001 & 0.12 & 56.49 & 0.996 \\
& 15 Sept. & -0.0001 & 0.12 & 59.36 & 0.967 \\
& 19 Sept. & -0.0001 & 0.11 & 59.38 & 0.944 \\
& 22 Sept. & -0.0003 & 0.17 & 50.11 & 0.959 \\
& 25 Sept & -0.0002 & 0.11 & 47.62 & 0.928 \\
\hline
\end{tabular}

and higher hue angle values on most harvest dates and for both cultivars.

\section{Summary}

A summary of these four experiments indicates that ReTain consistently and effectively delayed fruit harvest by about 3 to $4 \mathrm{~d}$. This response was rate dependent and increased in a linear fashion with increasing rates of AVG.

These data are in agreement with the results of others on peaches (Belding and Lokaj, 2002; Dekazos, 1981; Jobling et al., 2003; Kim et al., 2004; Legendre et al., 2003, Vizzotto et al., 2002) and nectarines (Rath and Prentice, 2004). AVG also increased fruit firmness in a rate dependent fashion even though fruit were harvested at similar visual stages of maturity (based on uniform background color). The positive influence of increasing concentrations of AVG on firmness has not been observed in previous studies (Kim, et al., 2004; Vizzotto et al., 2002), although when compared with untreated trees, AVG has been reported elsewhere to either improve peach fruit firmness or delay softening (Bregoli etal, 2001, 2002; Rath and Prentice, Singh et al., 2003a, 2003b; Vizzotto et al., 2002).

In contrast to previous studies on peaches (Kim et al., 2004; Vizzotto et al., 2002) the present study failed to show that AVG will delay or reduce preharvest fruit drop even though accumulated fruit drop by the last harvest date from untreated control trees ranged from $16 \%$ to $18 \%$ for 'Venture' and 'Babygold 7 '. In 2003 , trees were exposed to wind gust up to $55 \mathrm{~km} \cdot \mathrm{h}^{-1}$ (from the tail end of a tropical low pressure system) for the period $72 \mathrm{~h}$ before and including the last harvest date on 25 Sept., and although anticipated, no statistical differences in fruit drop between AVG or surfactant treatments was detected, even for the preharvest drop susceptible cultivar 'Babygold 7'. One explanation for this may be because 80 and $90 \%$ of untreated fruit from the 'Babygold 7' and 'Venture' trees, respectively, had already been harvested before this event. Where preharvest drop is a concern commercially, the cling processing cultivars are generally harvested over two picking dates and greater differences in fruit drop between untreated and AVG treated trees might occur if a greater percentage of fruit remains unharvested before the final harvest date. Greater fruit retention on AVG treated peaches has been observed in other studies (Kim et al., 2004; Vizzotto et al., 2002)

In contrast to a study on 'Feichen' peaches by Ju et al (1999), this study failed to demonstrate any measurable effect on increased yield in either of the four experiments. The delay in harvest was perhaps insufficient for fruit to accumulate additional assimilates which could conceivably translate into greater yields and mean fruit size. Only in one instance in 2003, was the mean fruit size of 'Babygold 7' increased, however this response, was more likely an indirect effect of crop load. In a larger scale experiment in Australia on 'Arctic Snow' nectarine, a $12 \%$ increase in yield and greater fruit size was realized from $\mathrm{AVG}$-treated trees, primarily a result of a reported 2.75 day delay 
in maturity (Rath and Prentice, 2004).

The surfactants used in these experiments had little to no effect on the efficacy of AVG. AVG applied with or without a surfactant equally and effectively both delayed maturity and increased fruit firmness. Initially it was anticipated that surfactants might aid in surface wetting of the fruit, but visual observations at the time of spraying indicated that spray mixtures in the absence of surfactants easily penetrated, and perhaps were aided by, the extensive hairy surface of the peach fruit. In fact peach fuzz may help AVG and other growth regulator sprays persist longer on the fruit surface had they otherwise not been present.

The most notable influence of surfactants was that, in one instance, Regulaid and Li 700 advanced color development and in another, fruit treated with $\mathrm{Li}-700$ and AVG were firmer in comparison with fruit treated with Regulaid and $\mathrm{AVG}$.

In summary, these data indicate the advantage in AVG-treated fruit on fruit quality, however, because of relatively small sample sizes, and inherent fruit variation on the tree on in the orchard, larger orchard studies are required to fully evaluate the benefits of AVG on improved yield and fruit size. Furthermore, the benefits of AVG on local fresh-market cultivars grown in Canadian production regions requires evaluation.

\section{Literature Cited}

Autio, W.R. and W.J. Bramlage. 1982. Effects of AVG on maturation, ripening, and storage of apples. J. Amer. Soc. Hort. Sci. 107:974-1077.

Bangerth, F. 1978. The effect of a substituted amino acid on ethylene biosynthesis, respiration, ripening, and preharvest drop of apple fruit. J. Amer. Soc. Hort. Sci. 103:401-404.

Belding, R.D. and G.R.W. Lokaj. 2002. Aminoethoxyvinylglycine treatment of peach fruit reduces ethylene and softening. HortScience 37(7):1065-1068
Bregoli, A.M, E. Sabatini, and G. Costa. 2001. Preliminary results on the use of growth regulators to control fruit ripening in Redhaven peach. (Risultati preliminari sull'impiego di sostanze di crescita per il controllo della maturazione dei frutti di pesco Redhaven), p. 199-201. In: S. Sansavini (ed.). XXIV Convegno Peschicolo. Per una nuova peschicoltura: produzione, organizzazione, mercato, Cesena, Italia, 24-25 Feb. 2000.

Bregoli, A.M., S. Scaramagli, G. Costa, E Sabatini, V.Ziosi, S Biondi, and P. Torrigiani. 2002. Peach (Prunus persica) fruit ripening: Aminoethoxyvinylglycine (AVG) and exogenous polyamines affect ethylene emission and flesh firmness. Physiol. Plantarum 114 (3):472-481

Byers, R.E. 1997. Peach and nectarine fruit softening following aminoethoxyvinylglycine sprays and dips. HortScience 32(1):86-88

Crisosto, C.H., P.B. Lombard, and L.H. Fuchigami. 1987. Spring applications of Ethephon and ethylene inhibitors on bloom delay, fruit set, and yield in 'Redhaven' peach. Acta Hort. 201:195-201

Dekazos, E.D. 1981. Effect of aminoethoxyvinylglycine on bloom delay, fruit maturity, and quality of 'Loring' and 'Rio Oso Gem' peaches. HortScience 16(4):520-522

Garner, D., C.H. Crososto, and E. Otieza. 2001. Controlled atmosphere storage and Aminoethoxyvinylglycine postharvest dip delay postcold storage softening of 'Snow King' Peach. HortTechnology 11(4):598-602.

Greene, D.W. 2002. Preharvest drop control and maturity of 'Delicious' apples as affected by aminoethoxyvinylglycine (AVG). J. Tree Fruit Prod. 3:1-10.

Greene, D.W. and J.R. Schupp. 2004. Effect of aminoethoxyvinylglycine (AVG) on preharvest drop, fruit quality, and maturation of 'McIntosh' apples. II. Effect of timing and concentration relationships and spray volume. HortScience 39(5):1036-1041

Jobling, J., S.C. Morris, and A.C. Rath. 2003. The effects of ReTain ${ }^{\mathrm{TM}}(\mathrm{R})(\mathrm{AVG})$ on the postharvest storage life of plums, peaches, nectarines, apples and bananas, p. 132-133. Australian postharvest horticulture conference, Brisbane, Australia, 1-3 Oct., 2003.

Ju, Z-G., Y-S. Duan, and Z-Q. Ju. 1999. Combina- tions of GA(3) and AVG delay fruit maturation, increase fruit size and improve storage life of 'Feicheng' peaches. J. Hort. Sci. Biotechnol. 74(5):579-583.

Kim, I.S., C.D. Choi, H.J. Lee, and J.K Byun. 2004. Effects of aminoethoxyvinylglycine on preharvest drop and fruit quality of 'Mibaekdo'peaches. S.-M.Kang (ed). Acta Hort. 653:173-178.

Legendre, L., A.C. Rath, and B. McGlasson. 2003. Preharvest application of AVG modifies harvest maturity and cool storage life of 'Arctic Snow' nectarines, $\mathrm{p} 227$. Australian postharvest horticulture conference, Brisbane, Australia, 1-3 Oct. 2003.

McGuire, R. 1992. Reporting of objective color measurements. HortScience 27(12):1254-1255.

Rath, A.C. and J. Prentice. 2004. Yield increase and higher flesh firmness of 'Arctic Snow' nectarines both at harvest in Australia and after export to Taiwan following preharvest application of ReTain ${ }^{\mathrm{TM}}$ plant growth regulator (aminoethoxyvinylglycine, AVG). Austral. J. Expt. Agr. 44(3):343-351

Schupp, J.R. And D.W. Greene. 2004. Effect of aminoethoxyvinylglycine (AVG) on preharvest drop, fruit quality, and maturation of 'McIntosh' apples. I. Concentration and timing of dilute applications of AVG. HortScience 39(5):1030-1035

Singh, Z., K. Kennison, and V. Agrez. 2003. Regulation of fruit firmness, maturity and quality of late maturing cultivars of peach with preharvest application of ReTain ${ }^{\mathrm{TM}}$. R.L. Prange (ed.). Acta Hort. 628(1):277-283

Stover, E., M.J. Fargione, C.B. Watkins, and K. A. Iungerman. 2003. Harvest management of Marshall 'McIntosh' apples: Effects of AVG, NAA, ethephon, and summer pruning on preharvest drop and fruit quality. HortScience 38(6): 1093-1099.

Tonutti, P., C. Bonghi, B. Ruperti, G.B. Tornielli, and A. Ramina 1977. Ethylene evolution and 1-aminocyclopropane-1-carboxylate oxidase gene expression during early development and ripening of peach fruit. J. Amer. Soc. Hort. Sci 122:642-647.

Vizzotto, G., E. Casatta, C. Bomben, A.M. Bregoli, E. Sabatini, and G. Costa. 2002. R.S. Johnson and C.H. Chrisosto (eds.). Peach ripening as affected by AVG. Acta Hort. 592:561-563 